\title{
Simultaneous Determination of 5 Flavonoids and 7 Saponins for Quality Control of Traditional Chinese Medicine Preparation Xinnaoshutong Capsule Using HPLC-VWD-ELSD
}

\author{
Jin Li, ${ }^{1}$ Yang Bai, ${ }^{1,2}$ Peng Zhang, ${ }^{1}$ Jun He, ${ }^{1,2}$ Jun Cao, ${ }^{3}$ Mingrui An, ${ }^{4}$ \\ Li-min Hu, ${ }^{1,2}$ Xiu-mei Gao, ${ }^{1}$ and Yan-Xu Chang ${ }^{1,2}$ \\ ${ }^{1}$ Tianjin State Key Laboratory of Modern Chinese Medicine, Tianjin University of Traditional Chinese Medicine, \\ Tianjin 300193, China \\ ${ }^{2}$ Tianjin Key Laboratory of Phytochemistry and Pharmaceutical Analysis, Tianjin University of Traditional Chinese Medicine, \\ Tianjin 300193, China \\ ${ }^{3}$ College of Material Chemistry and Chemical Engineering, Hangzhou Normal University, Hangzhou 310036, China \\ ${ }^{4}$ Department of Surgery, University of Michigan, Ann Arbor, MI 48109, USA
}

Correspondence should be addressed to Jun Cao; caojun91@163.com, Xiu-mei Gao; gaoxiumei@tjutcm.edu.cn, and Yan-xu Chang; tcmcyx@126.com

Received 15 August 2016; Accepted 10 November 2016; Published 16 January 2017

Academic Editor: Josep Esteve-Romero

Copyright (C) 2017 Jin Li et al. This is an open access article distributed under the Creative Commons Attribution License, which permits unrestricted use, distribution, and reproduction in any medium, provided the original work is properly cited.

\begin{abstract}
Xinnaoshutong capsule (XC) is a traditional Chinese prescription derived from the ripe fruit of Tribulus terrestris L. (TT). Although $\mathrm{XC}$ has long been considered as an important herbal medicine, no analytical method of marker compounds for quality assessment is registered in the Chinese Pharmacopoeia. A simple analytical method of twelve marker components was developed and validated by HPLC-VWD-ELSD method. Chromatographic separation by HPLC was carried out on a Hedera ODS 2 column $(4.6 \times$ $250 \mathrm{~mm}, 5 \mu \mathrm{m})$ by gradient elution with acetonitrile-water $(0.1 \%$ formic acid) as the mobile phase. Various extraction conditions were optimized to achieve twelve marker compounds with faster extraction and higher recovery. The analytical condition was then validated in terms of the linearity, accuracy and precision, repeatability, and stability. The twelve markers were successfully quantified in 30 batches of commercial samples. The developed HPLC-VWD-ELSD could be used as a rapid and reliable way in the assessment and quality control of XC and TT.
\end{abstract}

\section{Introduction}

Xinnaoshutong capsule (XC), a traditional Chinese herbal formula made from the aqueous extract of air-dried ripe fruit of Tribulus terrestris L. (TT), has been traditionally used to treat vascular diseases such as cerebral arteriosclerosis [1]. It has been proven that it possesses the pharmacological effect of activating blood circulation to dissipate blood stasis, support healthy energy, and prevent cerebral arteriosclerosis [2]. The major chemical constituents of XC included saponins (furostanol saponins) and flavonoids. Saponin possessed diverse pharmacological activities such as antifungal [3], antihyperlipidemic [4, 5], antihypertensive [6], antidepressive [7], anticerebral ischemia [8], and anti-UVB-induced damage effects [9]. It also increases hormonal levels [10, 11], improves erectile function [12], and repels the dengue fever mosquito [13] and it is cytotoxic against most cell lines from leukemia [14]. Meanwhile, flavonoids have also demonstrated tremendous anticancer, antioxidant, anti-inflammatory, antibacterial, and antifungal effects [15-18]. Thus, the clinical and pharmacological effect of XC may be exerted through the combination and interaction of the above two types of constituents. 
In order to guarantee XC to have an optimal therapeutic effect, the consistency of quality considered a crucial factor. This can be accomplished by maintaining a constant content of the bioactive compounds in $\mathrm{XC}$. There are numerous chemical compounds in XC and their complexity and diversity make it difficult to determine the contents of all compounds contained in it. In addition, the composition of constituent compounds can be easily affected by the manufacturing process. Therefore, it is necessary to consider multiple marker constituents in $\mathrm{XC}$ to evaluate its quality.

Several approaches to the quantitative analysis of constituent components have been reported for the quality assessment of TT. Most studies of the chemical quantification of TT are focused on the simultaneous determination of fewer than five compounds (i.e., diosgen [19], terrestroneoside [20], saponin D [21], protodioscin [22] quercetin, kaempferol, and isorhamnetin [23] and quercetin, kaempferol, isorhamnetin, hecogenin, and tigogenin [24]). However, there is no officially established quality control method for XC till now. Hence, variations in the amounts of constituent compounds in XC preparations from different batches cannot be investigated.

In recent years, high performance liquid chromatography-diode array detector-evaporative light scattering detector (HPLC-DAD-ELSD) method was widely used in detecting the low ultraviolet absorption compounds, which is a simple, rapid, and accurate method and can reflect variations in the content of constituent components in herbal preparations and has been widely used for quality assessment of traditional Chinese herbal medicines and their preparations [25-27]. For the purposes of the quality of XC, an HPLC-VWD-ELSD (high performance liquid chromatography-variable wavelength detector-evaporative light scattering detector) was developed and validated to quantify some components. Rutin (8), Isoquercitrin (9), kaempferol (10), quercetin (11), and isorhamnetin (12) are typical flavonoids [23, 24] while protodioscin (1) and Pseudoprotodioscin (3) are typical saponins of T. terrestris $[28,29]$. Till now, some publications on the isolation and structure elucidation of five saponins ((25R)-26-O- $\beta$-Dglucopyranosyl- $5 \alpha$-furostan-3 $\beta, 22 \alpha, 26$-triol-3-O- $\beta$-D-xylopyranosyl- $(1 \rightarrow 2)$ - $[\beta$-D-xylopyranosyl- $(1 \rightarrow 3)]-\beta$-D-glucopyranosyl- $(1 \rightarrow 4)$ - $[\alpha$-L-rhamnopyranosyl- $(1 \rightarrow 2)]-\beta$-D-galactopyranoside(2),26-O- $\beta$-D-glucopyranosyl-(25R)- $5 \alpha$-furostan-20(22)-en-3 $\beta, 26$-diol-3-O- $\{\beta$-D-xylopyranosyl- $(1 \rightarrow 2)$ $[\beta$-D-xylopyranosyl- $(1 \rightarrow 3)]-\beta$-D-glucopyranosyl- $(1 \rightarrow 4)-[\alpha$ L-rhamnopyranosyl- $(1 \rightarrow 2)]$ - $\beta$-D - galactopyranoside $\}(4)$, Terrestrinin A (5) and hecogenin-3-O- $\beta$-D-glucopyrano$\operatorname{syl}(1 \rightarrow 2)$ - $[\beta$-D-xylopyranosyl- $(1 \rightarrow 3)]-\beta$-D-glucopyranosyl$(1 \rightarrow 4)$ - $\beta$-D-galactopyranoside $(6)$ do not offer any information about contents of $T$. terrestris and its related preparations XC $[3,29,30]$. This makes the proper selection of the marker components impossible for quality control of XC. The aphrodisiac properties of 1 [31] and glycolate oxidase inhibitory activity of 10 and 11 are reported in the literature [32]. In order to clarify distribution of chemical components in $\mathrm{XC}$, these seven saponins and five flavonoids (Figure 1) were detected and selected as marker components for evaluating the consistency of quality of $\mathrm{XC}$ and TT.

In the present study, these twelve components were also simultaneously determined by developed and validated high performance liquid chromatography-variable wavelength detector-evaporative light scattering detector (HPLCVWD-ELSD). It was demonstrated that a feasible HPLCVWD-ELSD method is more reliable to determine sample contents as a means of developing quality control of TCM preparation.

\section{Experimental}

2.1. Reagents and Materials. XC (30 batches) were obtained from Jilin Aodong Pharmaceutical (Jilin, China). Reference compounds of (2) (25R)-26-O- $\beta$-D-glucopyranosyl- $5 \alpha$-furostan-3 $\beta, 22 \alpha, 26$-triol-3-O- $\beta$-D-xylopyranosyl- $(1 \rightarrow 2)-[\beta$ $\mathrm{D}$-xylopyranosyl- $(1 \rightarrow 3)]-\beta$-D-glucopyranosyl- $(1 \rightarrow 4)-[\alpha$-Lrhamnopyranosyl-(1 $\rightarrow 2)]-\beta$-D-galactopyranoside, (4) 26-O$\beta$-D-glucopyranosyl-(25R)- $5 \alpha$-furostan-20(22)-en-3 $\beta$,26diol-3-O- $\{\beta$-D -xylopyranosyl- $(1 \rightarrow 2)$ - $[\beta$-D-xylopyranosyl$(1 \rightarrow 3)]-\beta$-D-glucopyranosyl- $(1 \rightarrow 4)$ - $[\alpha$-L-rhamnopyranosyl$(1 \rightarrow 2)]-\beta$-D-galactopyranoside $\}$, (5) Terrestrinin A, and (6) hecogenin-3-O- $\beta$-D-glucopyranosyl $(1 \rightarrow 2)$ - $[\beta$-D-xylopyranosyl- $(1 \rightarrow 3)]$ - $\beta$-D-glucopyranosyl- $(1 \rightarrow 4)-\beta$-D-galactopyranoside were isolated from Tribulus terrestris L. in our laboratory. Reference compounds of (1) Protodioscin, (3) Pseudoprotodioscin, and (7) Tibllin were purchased from Zhongxin Innova (Tianjin, China), and (8) Rutin, (9) Isoquercitrin, (10) kaempferol, (11) quercetin, and (12) Isorhamnetin were purchased from Chengdu Musi Bio. Sci. and Tec. Co. Ltd. (Chengdu, China). The purity of each reference sample was determined to be above $98 \%$ by normalization of the peak area detected by HPLC-VWDELSD. Deionized water used for sample preparations and mobile phase was provided by a Milli-Q Academic ultrapure water system (Millipore, Bedford, MA, USA); acetonitrile and methanol were of HPLC grade (Tianjin Concord Science Co. Ltd., Tianjin, China). All other chemicals were of reagent grade.

\subsection{Sample Preparation}

2.2.1. Reference Standard Solutions. All standard compounds were individually dissolved with methanol (approximately $1.0 \mathrm{mg} \mathrm{mL}^{-1}$ ). The mixed stock solution of twelve reference compounds was prepared by dissolving accurately weighted portions of the standards in methanol at a stock concentration of $0.3 \mathrm{mg} \mathrm{mL}^{-1}$ for compounds $\mathbf{1}, \mathbf{3}$, and $\mathbf{6}$, $0.6 \mathrm{mg} \mathrm{mL}^{-1}$ for compound $2,2.1 \mathrm{mg} \mathrm{mL}^{-1}$ for compound 4 , $0.78 \mathrm{mg} \mathrm{mL}^{-1}$ for compound $5,0.24 \mathrm{mg} \mathrm{mL}^{-1}$ for compound 7 , and $0.015 \mathrm{mg} \mathrm{mL}^{-1}$ or all other compounds. The mixed standard solution containing all the compounds was further diluted to obtain the working standard solution with a series of concentrations. All the standard solutions were stored at $4^{\circ} \mathrm{C}$.

2.2.2. Sample Solution Preparation. A weight of $0.7 \mathrm{~g} \mathrm{XC}$ was accurately taken and then ultrasonically extracted for 

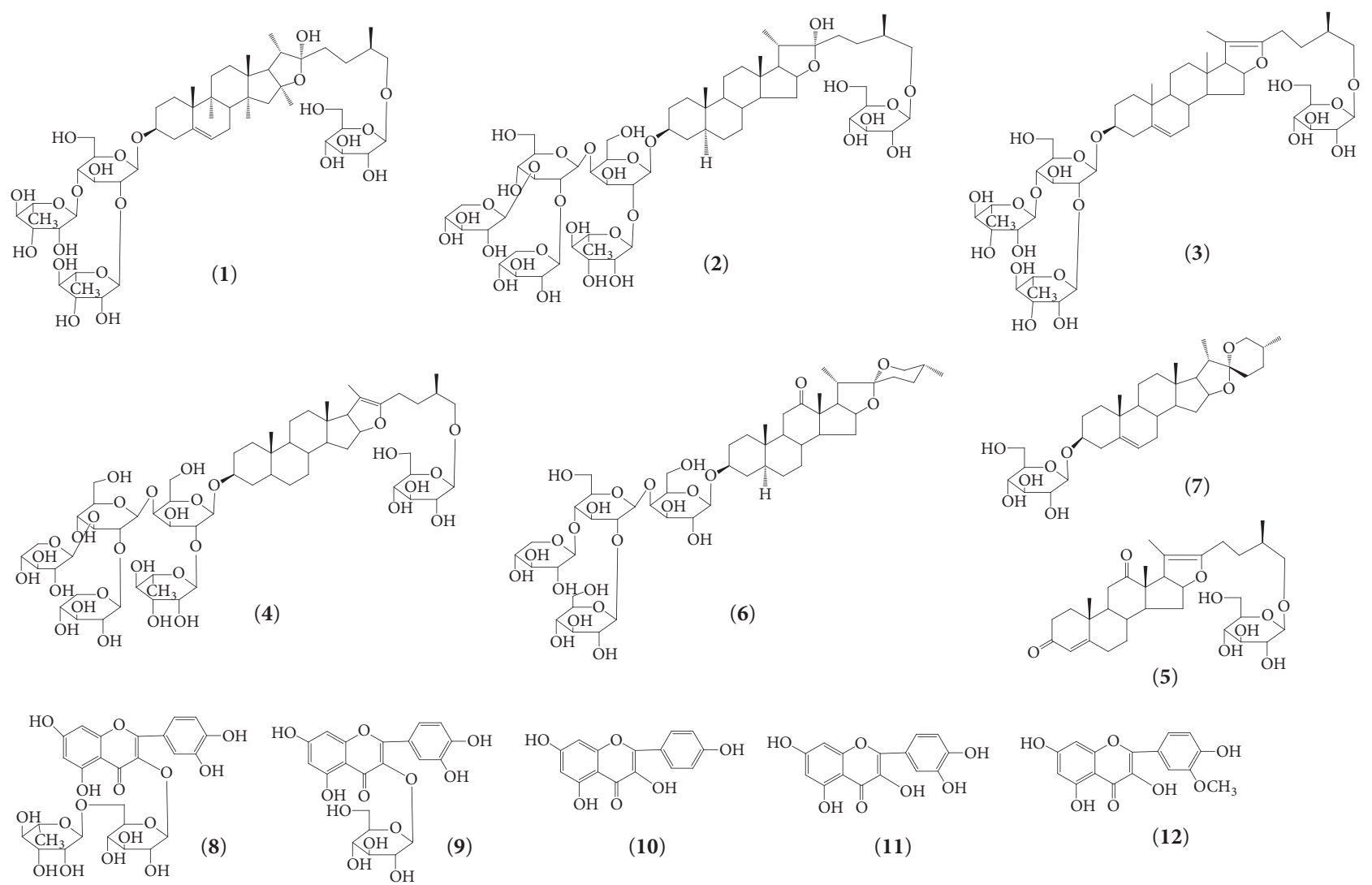

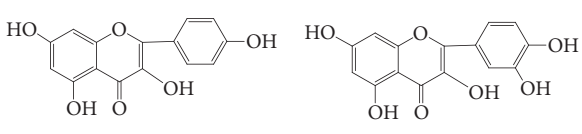

(10)

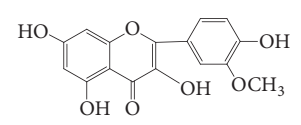

(12)

Figure 1: Chemical structures of the twelve bioactive components. (1) Protodioscin, (2) (25R)-26-O- $\beta$-D-glucopyranosyl-5 $\alpha$-furostan$3 \beta, 22 \alpha$,26-triol-3-O- $\beta$-D -xylopyranosyl-( $1 \rightarrow 2)$ - [ $\beta$-D-xylopyranosyl-( $1 \rightarrow 3)]$ - $\beta$-D-glucopyranosyl-( $1 \rightarrow 4)$ - $[\alpha$-L-rhamnopyranosyl- $(1 \rightarrow 2)]$ $\beta$-D-galactopyranoside, (3) Pseudoprotodioscin, (4) 26-O- $\beta$-D-glucopyranosyl-(25R)-5 $\alpha$-furostan-20(22)-en-3 $\beta$,26-diol-3-O- $\{\beta$-D-xylo-

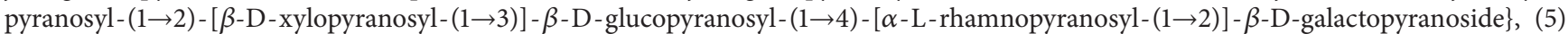
Terrestrinin A, (6) hecogenin-3-O- $\beta$-D-glucopyranosyl $(1 \rightarrow 2)$ - $[\beta$-D-xylopyranosyl- $(1 \rightarrow 3)]$ - $\beta$-D-glucopyranosyl- $(1 \rightarrow 4)$ - $\beta$-D-galactopyranoside, (7) Tibllin, (8) Rutin, (9) Isoquercitrin, (10) kaempferol, (11) quercetin, and (12) isorhamnetin.

40 min with $10 \mathrm{ml}$ pure methanol, respectively. The sample solution was centrifuged at $14,000 \mathrm{rpm}$ for $10 \mathrm{~min}$ and then an aliquot of the solutions was used for analysis, respectively.

2.2.3. Instrument and Chromatographic Conditions. All experiments were performed on an Agilent 1200 HPLC system (Agilent Corp., USA) which consisted of a vacuum degasser, a binary pump, an auto sampler, a column compartment, and a VWD coupled with an ELSD (Alltech Associates, Deerfield, USA). Data acquisition and processing were operated by ChemStation software (Agilent Technologies, USA). The drift tube temperature for ELSD was $70^{\circ} \mathrm{C}$, and the nebulizing gas flow rate was $1.8 \mathrm{~L} \mathrm{~min}^{-1}$. A constant flow rate of $1.0 \mathrm{mLmin}^{-1}$ was employed throughout the analysis. All analysis was performed at $30^{\circ} \mathrm{C}$. The chromatographic separations were achieved on a Hedera ODS 2 column $(250 \mathrm{~mm} \times 4.6 \mathrm{~mm}, 5 \mu \mathrm{m})$. The mobile phases consisted of $0.05 \%$ formic acid (A) and acetonitrile (B) with a gradient elution as follows: $0-5 \mathrm{~min}, 5-15 \%$ (B); $5-$ $10 \mathrm{~min}, 15-20 \%$ (B); $10-15 \mathrm{~min}, 20-20 \%$ (B); $15-20 \mathrm{~min}, 20-$ $23 \%$ (B); $20-27 \mathrm{~min}, 23-23 \%$ (B); $27-30 \mathrm{~min}, 23-26 \%$ (B); 30-45 min, 26-26\% (B); 45-50 min, 26-31\% (B); 50-55 min, $31-35 \%$ (B); $55-65 \mathrm{~min}, 35-35 \%$ (B); 65-70 $\mathrm{min}, 35-39 \%$ (B); 70-75 min, $39-39 \%$ (B); 75-80 min, $39-50 \%$ (B); $80-$ $85 \mathrm{~min}, 50-60 \%$ (B); 85-90 min, 60-90\% (B); 90-95 min, $90-$ $100 \%$ (B); $95-100 \mathrm{~min}, 100-100 \%$ (B). Then, a $10 \mu \mathrm{L}$ aliquot of the solution was injected into the HPLC-VWD-ELSD system for analysis. The detection wavelength was set at $360 \mathrm{~nm}$.

2.3. Method Validation. A stock solution, which contained 12 analytes, was prepared with methanol and diluted to a series of appropriate concentrations for the construction of calibration curves. The solutions were brought to room temperature and an aliquot of $10 \mu \mathrm{L}$ was injected into HPLC for analysis. For the seven saponins, the calibration curves were calculated by linear regression of the double logarithmic plots of the peak area obtained by ELSD detection versus the 


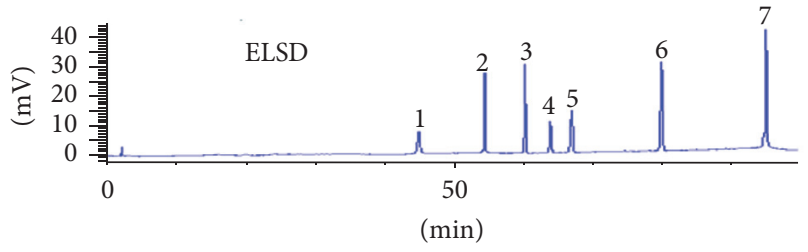

(a)

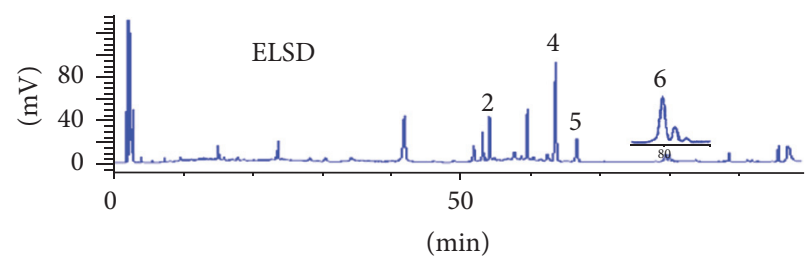

(c)

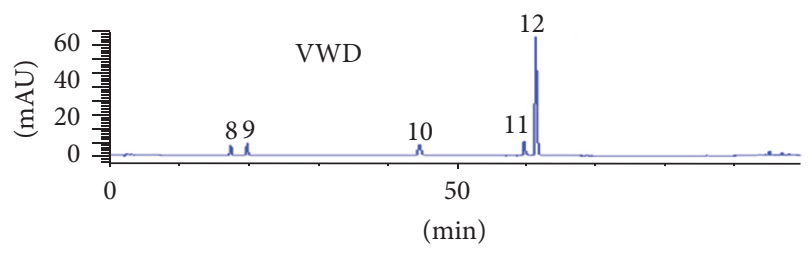

(b)

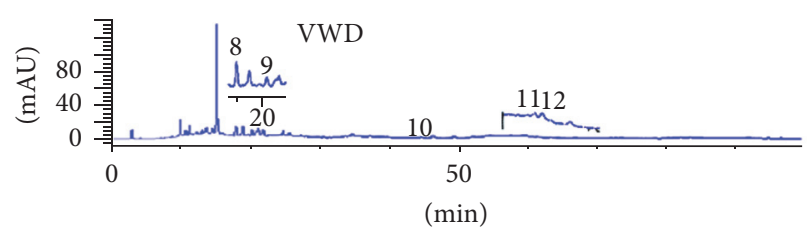

(d)

FIgURE 2: Typical HPLC-VWD-ELSD chromatograms for all of the reference compounds (a, b) and real sample (c, d). (1) Protodioscin, (2) (25R)-26-O- $\beta$-D -glucopyranosyl- $5 \alpha$-furostan-3 $\beta, 22 \alpha, 26$ - triol-3-O- $\beta$-D-xylopyranosyl- $(1 \rightarrow 2)$ - $[\beta$-D-xylopyranosyl- $(1 \rightarrow 3)]-\beta$-D-glucopyranosyl-(1 $\rightarrow 4)$-[ $\alpha$-L-rhamnopyranosyl- $(1 \rightarrow 2)]-\beta$-D-galactopyranoside, (3) Pseudoprotodioscin, (4) 26-O- $\beta$-D-glucopyranosyl- $(25 \mathrm{R})-5 \alpha$ furostan-20(22)-en-3 $\beta, 26$-diol-3-O- $\{\beta$-D-xylopyranosyl-( $\rightarrow 2)$-[ $\beta$-D-xylopyranosyl-( $1 \rightarrow 3)]$ - $\beta$-D-glucopyranosyl- $(1 \rightarrow 4)$ - $[\alpha$-L-rhamnopy-

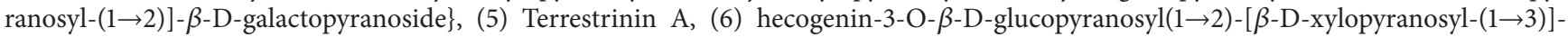
$\beta$-D-glucopyranosyl-(1 $\rightarrow 4)$ - $\beta$-D-galactopyranoside, (7) Tibllin, (8) Rutin, (9) Isoquercitrin, (10) kaempferol, (11) quercetin, and (12) isorhamnetin.

concentration of the reference solution injected. For the five flavonoids, the calibration curves were calculated by linear regression of the peak area obtained by UV detection versus the concentration of the reference solution injected. The limits of detections (LODs) and quantifications (LOQs) under the present chromatographic conditions were determined by diluting the standard solution when the signal-to-noise ratios $(\mathrm{S} / \mathrm{N})$ of analytes were about 3 and 10, respectively. The $\mathrm{S} / \mathrm{N}$ was calculated as the peak height divided by the background noise value. The analyses were performed six times on the same day. Recovery test was used to evaluate the accuracy of this method. The test was performed by adding accurately known amounts of the 12 standards into a certain amount $(0.35 \mathrm{~g})$ of XC separately. The spiked samples were then extracted, processed, and quantified in accordance with the methods mentioned above. Six replicates were performed for the test. The average recovery percentage was calculated by the formula: recovery $(\%)=$ (observed amount - original amount)/spiked amount $\times 100 \%$. Accuracy presented as percent recovery, whereas the repeatability was calculated as the RSD of the signal. Interday accuracy and intermediate precision were determined as the average values of five intraday measurements taken over three days' period. The stability test was performed with one real sample solution which was stored at room temperature and analyzed at 0,2 , $4,6,12$, and 24 hours.

\section{Results and Discussion}

3.1. Optimization of Extraction Procedure. In order to obtain an efficient extraction of flavonoids and saponins from XC, the variables of the extraction process were optimized. The different ultrasonical extraction time of
$20 \mathrm{~min}$, $40 \mathrm{~min}$, and $60 \mathrm{~min}$ was investigated. The results suggested that the amounts of flavonoids and saponins are no significant difference with the extraction time of $40 \mathrm{~min}$ and $60 \mathrm{~min}$ while both of them were higher than extraction time of $20 \mathrm{~min}$. Therefore, the optimum sample extraction condition was achieved by methanol for $40 \mathrm{~min}$.

3.2. Optimization of HPLC-VWD Conditions. The optimization of HPLC conditions was carried out by using the mixed reference compound solutions. Considering the polarity differences of twelve compounds, different kinds of gradient elution of acetonitrile-water and gradient elution of acetonitrilewater $(0 \%, 0.05 \%$, and $0.1 \%$ formic acid), methanol-water $(0 \%, 0.05 \%$, and $0.1 \%$ formic acid) were investigated for XC, respectively. The results showed that it could achieve better separation on the flavonoid compounds with gradient elution of acetonitrile- $0.05 \%$ formic acid aqueous solution. Then, nebulizing gas flow rate and evaporating temperature were optimized which were the two major instrument adjustments available for maximizing the detector response efficiency. Varying gas flow rates of $1.6,1.8$, and $2.0 \mathrm{Lmin}^{-1}$ were investigated. The results revealed that the noise was decreased when the flow rate increased. However, the responses would be weakened following the increased flow rate. Thus, a moderate flow rate of $1.8 \mathrm{~L} \mathrm{~min}^{-1}$ was adopted to achieve the better performance. With respect to the drift tube temperature, a high baseline noise at low temperature, the optimal drift tube temperature, was determined at $70^{\circ} \mathrm{C}$ according to the data computed with the ELSD software. Typical HPLC-VWD-ELSD chromatograms for all of the reference compounds and real samples are shown in Figures 2(a) and 2(b). 
TABLE 1: Linear regression data, LODs, and LOQs for the twelve compounds.

\begin{tabular}{|c|c|c|c|c|c|}
\hline Compounds & Linear regression $^{\mathrm{a}}$ & Linear range $\left(\mu \mathrm{g} \cdot \mathrm{mL}^{-1}\right)$ & $r^{2}$ & $\mathrm{LOD}^{\mathrm{b}}\left(\mu \mathrm{g} \cdot \mathrm{mL}^{-1}\right)$ & $\mathrm{LOQ}^{\mathrm{c}}\left(\mu \mathrm{g} \cdot \mathrm{mL}^{-1}\right)$ \\
\hline 1 & $y=1.6259 x+3.3728$ & $50-300$ & 0.9993 & 3.3 & 10 \\
\hline 2 & $y=1.5654 x+3.39$ & $40-600$ & 0.9981 & 1.3 & 4 \\
\hline 3 & $y=1.6565 x+3.5949$ & $50-300$ & 0.999 & 3.3 & 10 \\
\hline 4 & $y=1.554 x+3.0474$ & $70-2100$ & 0.995 & 2.3 & 7 \\
\hline 5 & $y=1.5726 x+3.2295$ & $52-780$ & 09956 & 4.3 & 13 \\
\hline 6 & $y=1.8064 x+3.6226$ & $50-300$ & 0.9985 & 3.3 & 10 \\
\hline 7 & $y=1.3249 x+3.6714$ & $16-240$ & 0.9983 & 0.64 & 2 \\
\hline 8 & $Y=17419 X-1.361$ & $0.5-15$ & 0.9962 & 0.13 & 0.5 \\
\hline 9 & $Y=19853 X+2.8905$ & $0.5-15$ & 0.9997 & 0.13 & 0.25 \\
\hline 10 & $Y=30126 X+3.3194$ & $0.5-15$ & 0.9993 & 0.13 & 0.5 \\
\hline 11 & $Y=43107 X-1.3252$ & $0.15-15$ & 0.9999 & 0.13 & 0.25 \\
\hline 12 & $Y=29297 X+0.2176$ & $0.15-15$ & 0.9999 & 0.15 & 0.25 \\
\hline
\end{tabular}

${ }^{a} Y$ is the peak area in UV chromatograms monitored at $360 \mathrm{~nm}, X$ is the compound concentration injected, and $y$ and $x$ are the logarithmic values of area and concentration injected in ELSD chromatograms.

${ }^{\mathrm{b}} \mathrm{LOD}$ refers to the limits of detection.

${ }^{c} \mathrm{LOQ}$ refers to the limits of quantification.

TABLE 2: Intra- and interday accuracy, precision, and stability for the twelve compounds.

\begin{tabular}{|c|c|c|c|c|c|c|}
\hline \multirow{2}{*}{ Components } & \multirow{2}{*}{ Concentration $\left(\mu \mathrm{g} \cdot \mathrm{mL}^{-1}\right)$} & \multicolumn{2}{|c|}{ Intraday } & \multicolumn{2}{|c|}{ Interday } & \multirow{2}{*}{$\begin{array}{c}\text { Stability } \\
\text { Precision (RSD, \%) }\end{array}$} \\
\hline & & Accuracy $^{\mathrm{a}}(\%)$ & Precision (RSD, \%) & Accuracy (\%) & Precision (RSD, \%) & \\
\hline 1 & 0.1158 & 105 & 2.42 & 95.7 & 2.17 & 1.23 \\
\hline 2 & 0.212 & 95.0 & 2.12 & 97.0 & 1.80 & 1.74 \\
\hline 3 & 0.0947 & 110 & 1.67 & 95.9 & 2.66 & 2.52 \\
\hline 4 & 1.6225 & 108 & 3.71 & 87.4 & 2.86 & 0.93 \\
\hline 5 & 0.4594 & 113 & 1.39 & 97.1 & 1.37 & 1.81 \\
\hline 6 & 0.2641 & 102 & 1.85 & 98.6 & 2.41 & 2.12 \\
\hline 7 & 0.0324 & 111 & 4.54 & 100 & 2.90 & 2.67 \\
\hline 8 & 0.0171 & 102 & 2.07 & 90.1 & 1.66 & 0.32 \\
\hline 9 & 0.0060 & 110 & 2.58 & 88.3 & 2.54 & 0.59 \\
\hline 10 & 0.001 & 104 & 4.77 & 110 & 1.31 & 1.18 \\
\hline 11 & 0.0004 & 105 & 3.30 & 100 & 2.37 & 1.02 \\
\hline 12 & 0.0019 & 109 & 3.64 & 105 & 1.04 & 1.61 \\
\hline
\end{tabular}

${ }^{\mathrm{a}}$ Recovery $(\%)=($ amount determined - amount original)/amount spiked $\times 100 \%$.

3.3. Method Validation. The validation was performed following the requirements of the ICH Harmonized Tripartite Guideline. Some parameters including specificity, calibration range, linearity, sensitivity (LOD and LOQ), accuracy, repeatability, and intermediate precision were evaluated. The method was validated using real samples.

Linear regression of the twelve compounds showed good linear regression (all correlation coefficients $>0.99$ ) within the ranges of concentrations (Table 1). The LOD and the LOQ were less than 0.15 and $0.5 \mu \mathrm{gmL}^{-1}$ for flavonoids in VWD and were less than 4.3 and $13 \mu \mathrm{gmL}^{-1}$ for saponins in ELSD, respectively.

Intraday accuracy and repeatability were tested for twelve bioactive components. The solutions were prepared by adding known quantities of the analytes to the real samples. The analyses were performed six times on the same day. Accuracy presented as percent recovery, whereas the repeatability was calculated as the RSD of the signal. Interday accuracy and intermediate precision were determined as the average values of five intraday measurements taken over three days' period. Results are expressed in Table 2. Adequate recoveries (87.4$111 \%)$ and high precision $(<4.60 \%)$ of the signal were found, thus demonstrating the reliability of the twelve bioactive components contents in XC determined by the present analytical method. For stability, no significant diminution of the peak area was detected for all analytes in $24 \mathrm{~h}$ period.

3.4. Sample Analysis. The developed HPLC-VWD-ELSD method was applied for simultaneous determination of twelve bioactive components in thirty batches of XC and two batches of TT and the results are presented in Table 3. The typical chromatograms of the samples detected by VWD and ELSD, respectively, are shown in Figures 2(c) 


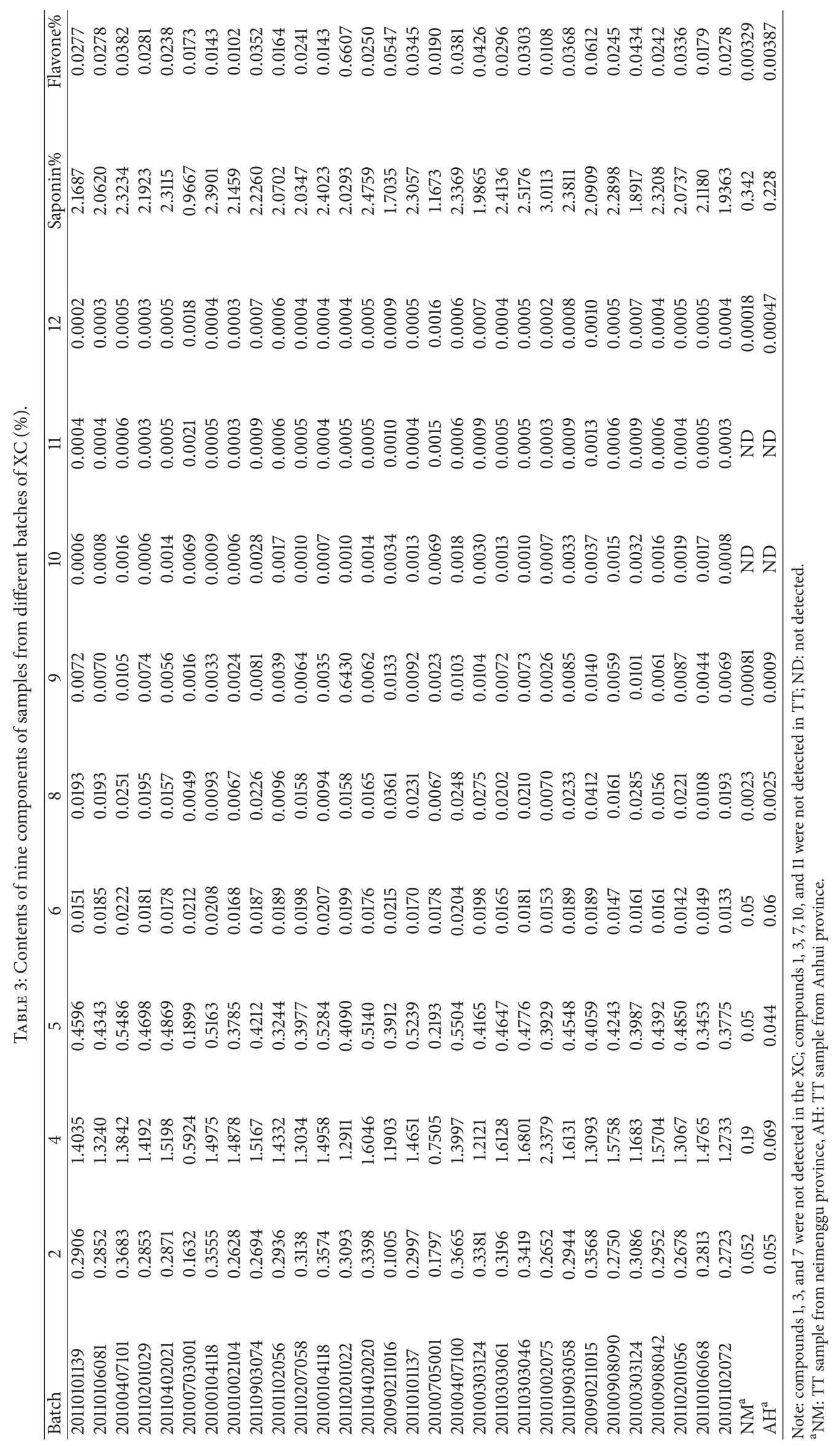


and 2(d). As can be seen from Table 3, it was found that compounds 1 (Protodioscin), 3 (Pseudoprotodioscin), and 7 (Tibllin) were not detected in the XC and compounds 1 (Protodioscin), 3 (Pseudoprotodioscin), 7 (Tibllin), 10 (kaempferol), and 11 (quercetin) were not detected in TT. The content of compound 4 (26-O- $\beta$-D-glucopyranosyl(25R)- $5 \alpha$-furostan-20(22)-en-3 $\beta, 26$-diol-3-O- $\{\beta$-D-xylopyranosyl- $(1 \rightarrow 2)$ - $[\beta$-D-xylopyranosyl- $(1 \rightarrow 3)]-\beta$-D-glucopyranosyl- $(1 \rightarrow 4)$ - $[\alpha$-L-rhamnopyranosyl- $(1 \rightarrow 2)]-\beta$-D-galactopyranoside\}) was significantly higher than other saponins in XC. Its content was (1.4\%) higher than half of the total saponin $(2.1 \%)$. The mean proportion of $26-\mathrm{O}-\beta-\mathrm{D}$ glucopyranosyl-(25R)- $5 \alpha$-furostan-20(22)-en-3 $\beta, 26$-diol-33 -O- $\{\beta$-D-xylopyranosyl- $(1 \rightarrow 2)-[\beta$-D-xylopyranosyl- $(1 \rightarrow 3)]$ $\beta$-D-glucopyranosyl-( $1 \rightarrow 4)$ - $[\alpha$-L-rhamnopyranosyl- $(1 \rightarrow 2)]$ $\beta$-D-galactopyranoside that accounted for the total saponins was 65\%. Similarly, content compound 8 (Rutin) and compound 9 (Isoquercitrin) were higher than other flavonoids in XC and TT. The mean proportion which contributed to the total flavonoids was 93.5\%. For these consequences, the three compounds can be seen as the index components of quality control of XC and TT.

It has been reported that protodioscin (compound 1) was converted to 22-O-methylprotodioscin in methanol rapidly [28]. In our experiment, methanol was chosen as the solvent to dissolve the standard sample and XC samples, so peak 1 is $22-\mathrm{O}$-methylprotodioscin in the standard solution and sample solutions. There also has the same situation on (25R)-26-O- $\beta$-D-glucopyranosyl- $5 \alpha$-furostan- $3 \beta, 22 \alpha$,26triol-3-O- $\beta$-D-xylopyranosyl- $(1 \rightarrow 2)$ - $[\beta$ - D - xylopyranosyl$(1 \rightarrow 3)]-\beta$-D-glucopyranosyl- $(1 \rightarrow 4)$ - $[\alpha$-L-rhamnopyranosyl$(1 \rightarrow 2)]-\beta$-D-galactopyranoside (compound 2$)$, we also use compound 2 to quantity methylated product. It was found that both 22-O-methylprotodioscin and protodioscin were not detected while compound 2 ranged from $0.1005 \%$ to $0.3683 \%$ in XC samples.

\section{Conclusion}

A reliable and simple method with HPLC-VWD-ELSD has been established and validated for simultaneous determination of twelve compounds in XC. The results of method validation illustrated that the presented method was precise, accurate, and sensitive as a practical technique for quantitative determination of multiple active components in XC. Twelve bioactive components including seven saponins and five flavonoids were selected as the chemical markers of XC to evaluate their quality of different batch samples. Compared with traditional HPLC-UV method, HPLCVWD-ELSD could solve the problem of low UV absorption of saponins in order to achieve determination of multiple active components. Compared with the previous quality control methods of spectrophotometry, this method was more accurate, sensitive, and stable. Consequently, discrimination should be part of the quality control for TCMs. The proposed method could be readily utilized as a routine analysis and effective tool to evaluate the quality control of TCMs.

\author{
Abbreviations \\ HPLC-VWD-ELSD: High performance liquid \\ chromatography-variable wavelength \\ detector-evaporative light scattering \\ detector \\ XC: $\quad$ Xinnaoshutong Capsule \\ RSD: $\quad$ Relative standard deviations \\ TCMs: Traditional Chinese medicines \\ LOD: Limit of detection \\ LLOQ: $\quad$ Lower limit of quantification.
}

\section{Competing Interests}

The authors declare that there is no conflict of interests regarding the publication of this paper.

\section{Authors' Contributions}

Yang Bai contributes equally as the first author in this study.

\section{Acknowledgments}

This research was supported by National Science and Technology Major Projects for Major New Drugs Innovation and Development (2010ZX09201-201, 2014ZX09304307-001005, and 2014ZX09201022-004), National Natural Science Foundation of China (81503213 and 81374050), and PCSIRT (IRT-14R41).

\section{References}

[1] National Pharmacopoeia Committee, Pharmacopoeia of People Republic of China, part 1, Chemical Industry Press, Beijing, China, 2010.

[2] J. Zhang, Y.-L. Zhang, J.-L. Lou et al., "Protective effects of Xinnao Shutong capsule on acute cerebral ischemic injury of multiple infarcts in rats," China Journal of Chinese Materiamedica, vol. 31, no. 23, pp. 1979-1982, 2006.

[3] J.-D. Zhang, Z. Xu, Y.-B. Cao et al., "Antifungal activities and action mechanisms of compounds from Tribulus terrestris L.," Journal of Ethnopharmacology, vol. 103, no. 1, pp. 76-84, 2006.

[4] M. Altug Tuncer, B. Yaymaci, L. Sati et al., "Influence of Tribulus terrestris extract on lipid profile and endothelial structure in developing atherosclerotic lesions in the aorta of rabbits on a high-cholesterol diet," Acta Histochemica, vol. 111, no. 6, pp. 488-500, 2009.

[5] T. Wang, R. C. Y. Choi, J. Li et al., "Antihyperlipidemic effect of protodioscin, an active ingredient isolated from the rhizomes of dioscorea nipponica," Planta Medica, vol. 76, no. 15, pp. 16421646, 2010.

[6] O. A. Phillips, K. T. Mathew, and M. A. Oriowo, "Antihypertensive and vasodilator effects of methanolic and aqueous extracts of Tribulus terrestris in rats," Journal of Ethnopharmacology, vol. 104, no. 3, pp. 351-355, 2006.

[7] Z. Wang, D. Zhang, S. Hui, Y. Zhang, and S. Hu, "Effect of tribulus terrestris saponins on behavior and neuroendocrine in chronic mild stress depression rats," Journal of Traditional Chinese Medicine, vol. 33, no. 2, pp. 228-232, 2013. 
[8] E.-P. Jiang, H. Li, J.-G. Chen, and S.-J. Yang, "Protection by the gross saponins of Tribulus terrestris against cerebral ischemic injury in rats involves the NF- $\kappa \mathrm{B}$ pathway," Acta Pharmaceutica Sinica B, vol. 1, no. 1, pp. 21-26, 2011.

[9] M. Sisto, S. Lisi, M. D’Amore et al., "Saponins from Tribulus terrestris L. protect human keratinocytes from UVB-induced damage," Journal of Photochemistry and Photobiology B: Biology, vol. 117, pp. 193-201, 2012.

[10] K. Gauthaman and A. P. Ganesan, "The hormonal effects of Tribulus terrestris and its role in the management of male erectile dysfunction - an evaluation using primates, rabbit and rat," Phytomedicine, vol. 15, no. 1-2, pp. 44-54, 2008.

[11] K. Hu and X. S. Yao, "Protodioscin (NSC-698 796): its spectrum of cytotoxicity against sixty human cancer cell lines in an anticancer drug screen panel," Planta Medica, vol. 68, no. 4, pp. 297-301, 2002.

[12] K. Gauthaman, A. P. Ganesan, and R. N. V. Prasad, "Sexual effects of puncturevine (Tribulus terrestris) extract (protodioscin): an evaluation using a rat model," Journal of Alternative and Complementary Medicine, vol. 9, no. 2, pp. 257-265, 2003.

[13] T. M. Y. El-Sheikh, Z. I. A. Al-Fifi, and M. A. Alabboud, "Larvicidal and repellent effect of some Tribulus terrestris L., (Zygophyllaceae) extracts against the dengue fever mosquito, Aedes aegypti (Diptera: Culicidae)," Journal of Saudi Chemical Society, vol. 20, no. 1, pp. 13-19, 2016.

[14] J. Do, S. Choi, J. Choi, and J. S. Hyun, "Effects and mechanism of action of a Tribulus terrestris extract on penile erection," Korean Journal of Urology, vol. 54, no. 3, pp. 183-188, 2013.

[15] S. H. Jung, B. J. Kim, E. H. Lee, and N. N. Osborne, "Isoquercitrin is the most effective antioxidant in the plant Thuja orientalis and able to counteract oxidative-induced damage to a transformed cell line (RGC-5 cells)," Neurochemistry International, vol. 57, no. 7, pp. 713-721, 2010.

[16] W. Lee, S.-K. Ku, and J.-S. Bae, "Barrier protective effects of rutin in LPS-induced inflammation in vitro and in vivo," Food and Chemical Toxicology, vol. 50, no. 9, pp. 3048-3055, 2012.

[17] P. R. Verma, S. A. Deshpande, Y. N. Kamtham, and L. B. Vaidya, "Hypolipidemic and antihyperlipidemic effects from an aqueous extract of Pachyptera hymenaea (DC.) leaves in rats," Food Chemistry, vol. 132, no. 3, pp. 1251-1257, 2012.

[18] D. D. Orhan, B. Özçelik, S. Özgen, and F. Ergun, "Antibacterial, antifungal, and antiviral activities of some flavonoids," Microbiological Research, vol. 165, no. 6, pp. 496-504, 2010.

[19] X. H. Yu, "Determination of diosgen in hydrolyzed from total saponins of Tribulusterrestris L. by ELSD -HPLC method," West China Journal of Pharmaceutical Sciences, vol. 23, pp. 474-475, 2008.

[20] Z. W. Guo, L. Zhang, and W. Li, "HPLC-ELSD determination of terrestroneoside in Tribulus terrestris L," Chinese Journal of Pharmaceutical Analysis, vol. 24, pp. 284-286, 2004.

[21] S. X. Xie, D. Han, H. F. Zhao, Y. S. Si, Y. J. Xu, and D. M. Xu, "Chinese Traditional Patent Medicine. Detemination of saponin D content in Tribulu sterrestris by HPLC-ELSD," Chinese Traditional Patent Medicine, vol. 31, pp. 434-436, 2009.

[22] M. Ganzera, E. Bedir, and I. A. Khan, "Determination of steroidal saponins in Tribulus terrestris by reversed-phase highperformance liquid chromatography and evaporative light scattering detection," Journal of Pharmaceutical Sciences, vol. 90, no. 11, pp. 1752-1758, 2001.

[23] Q. Shi, B. Y. Yu, L. S. Xu, and G. J. Xu, "Determination of three hydrolytic flavonoid aglycones in Tribulus terrestris and atriplexcentralasiatica by RP -HPLC," Chinese Journal of Pharmaceutical Analysis, vol. 19, pp. 75-77, 1999.

[24] H. J. Fang, K. S. Bi, Z. Z. Qian et al., "HPLC-DAD-ELSD determination of five active components in Tribulus terrestris L.Chinese," Journal of Pharmaceutical Analysis, vol. 32, article 6, 2012.

[25] F. Zhu, X. Jia, Y. Cai, and Y. Chen, "Quality evaluation of Yanghuo Sanqi tablet through a simultaneous determination of five major active Flavonoids and three main saponins by HPLCDAD-ELSD," Acta Chromatographica, vol. 24, no. 4, pp. 667677, 2012.

[26] B.-S. Sun, L.-J. Gu, Z.-M. Fang et al., "Simultaneous quantification of 19 ginsenosides in black ginseng developed from Panax ginseng by HPLC-ELSD," Journal of Pharmaceutical and Biomedical Analysis, vol. 50, no. 1, pp. 15-22, 2009.

[27] Q.-T. Yu, L.-W. Qi, P. Li, L. Yi, J. Zhao, and Z. Bi, "Determination of seventeen main flavonoids and saponins in the medicinal plant Huang-qi (Radix-Astragali) by HPLC-DAD-ELSD," Journal of Separation Science, vol. 30, no. 9, pp. 1292-1299, 2007.

[28] M. Ganzera, E. Bedir, and I. A. Khan, "Determination of steroidal saponins in Tribulus terrestris by reversed-phase high-performance liquid chromatography and evaporative light scattering detection," Journal of Pharmaceutical Sciences, vol. 90, no. 11, pp. 1752-1758, 2001.

[29] D. Dinchev, B. Janda, L. Evstatieva, W. Oleszek, M. R. Aslani, and I. Kostova, "Distribution of steroidal saponins in Tribulus terrestris from different geographical regions," Phytochemistry, vol. 69, no. 1, pp. 176-186, 2008.

[30] J. Wang, X. Zu, and Y. Jiang, "Five furostanol saponins from fruits of Tribulus terrestris and their cytotoxic activities," Natural Product Research, vol. 23, no. 15, pp. 1436-1444, 2009.

[31] K. Gauthaman, P. G. Adaikan, and R. N. V. Prasad, "Aphrodisiac properties of Tribulus Terrestris extract (Protodioscin) in normal and castrated rats," Life Sciences, vol. 71, no. 12, pp. 13851396, 2002.

[32] A. Shirfule, A. Sangamwar, and C. Khobragade, "Bioassayguided isolation and molecular ligand-target insight of a glycolate oxidase inhibitor from Tribulus terrestris L.", New Biotechnology, vol. 25, supplement, p. S319, 2009. 

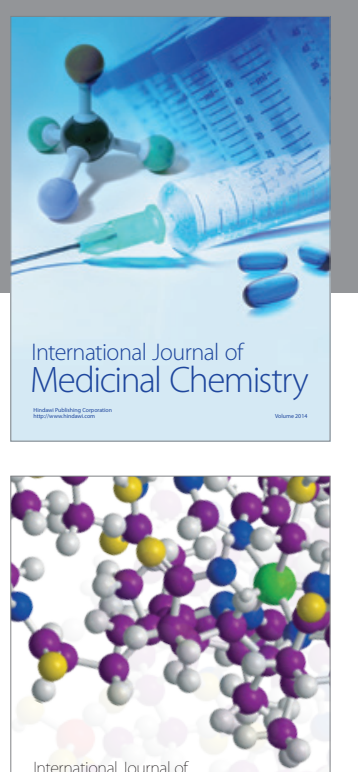

Carbohydrate Chemistry

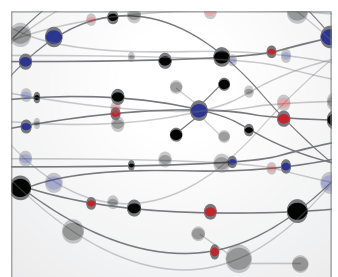

The Scientific World Journal
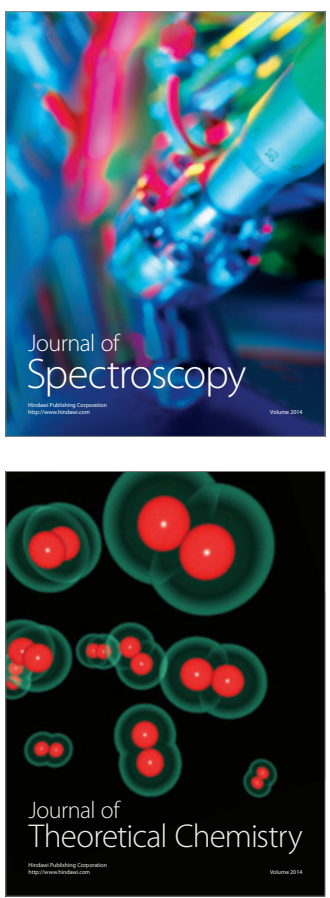
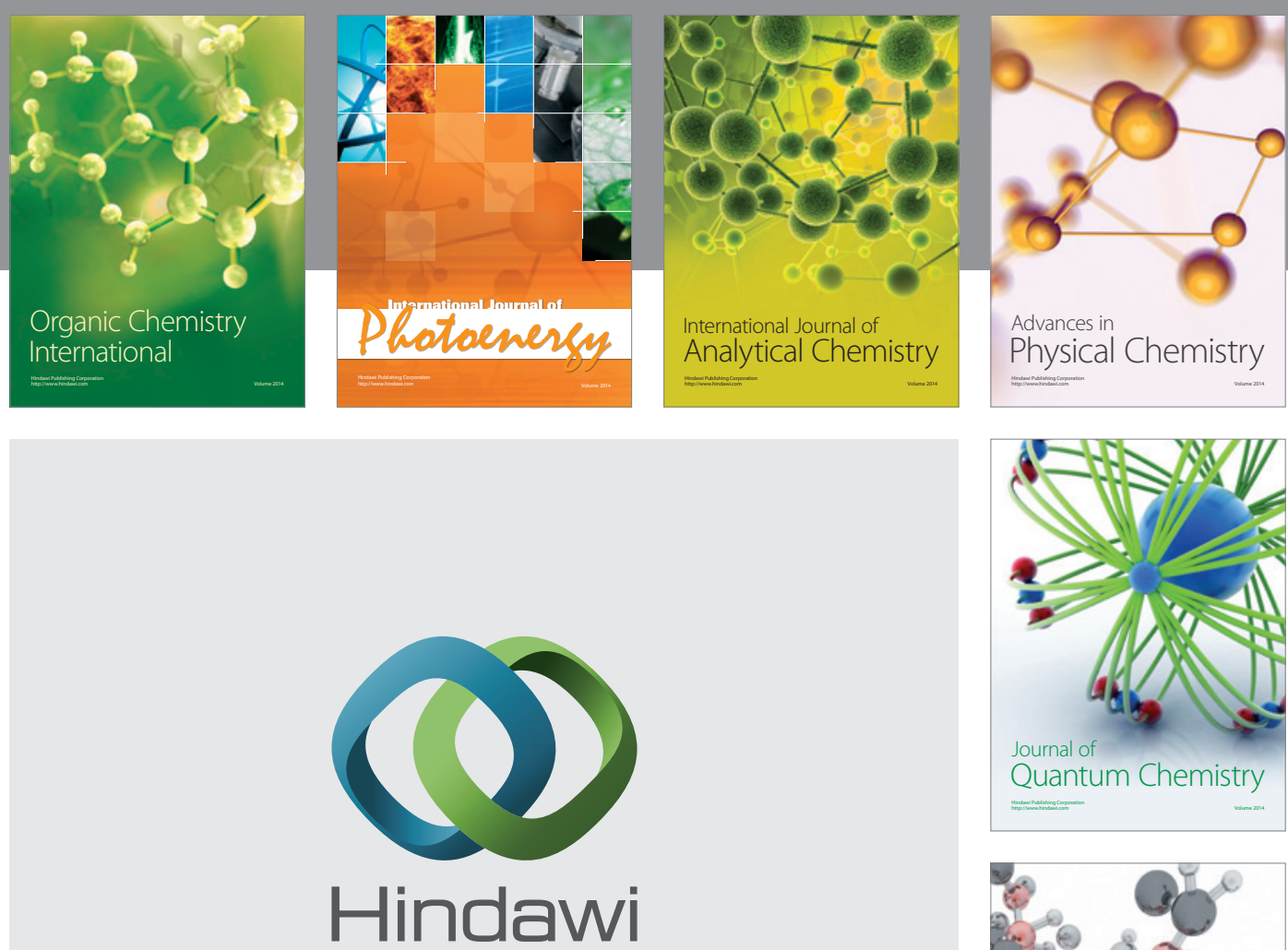

Submit your manuscripts at

https://www.hindawi.com

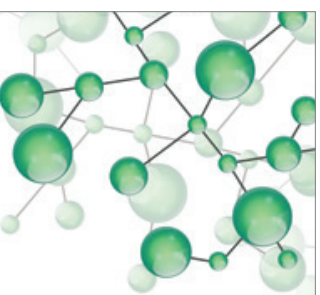

International Journal of

Inorganic Chemistry
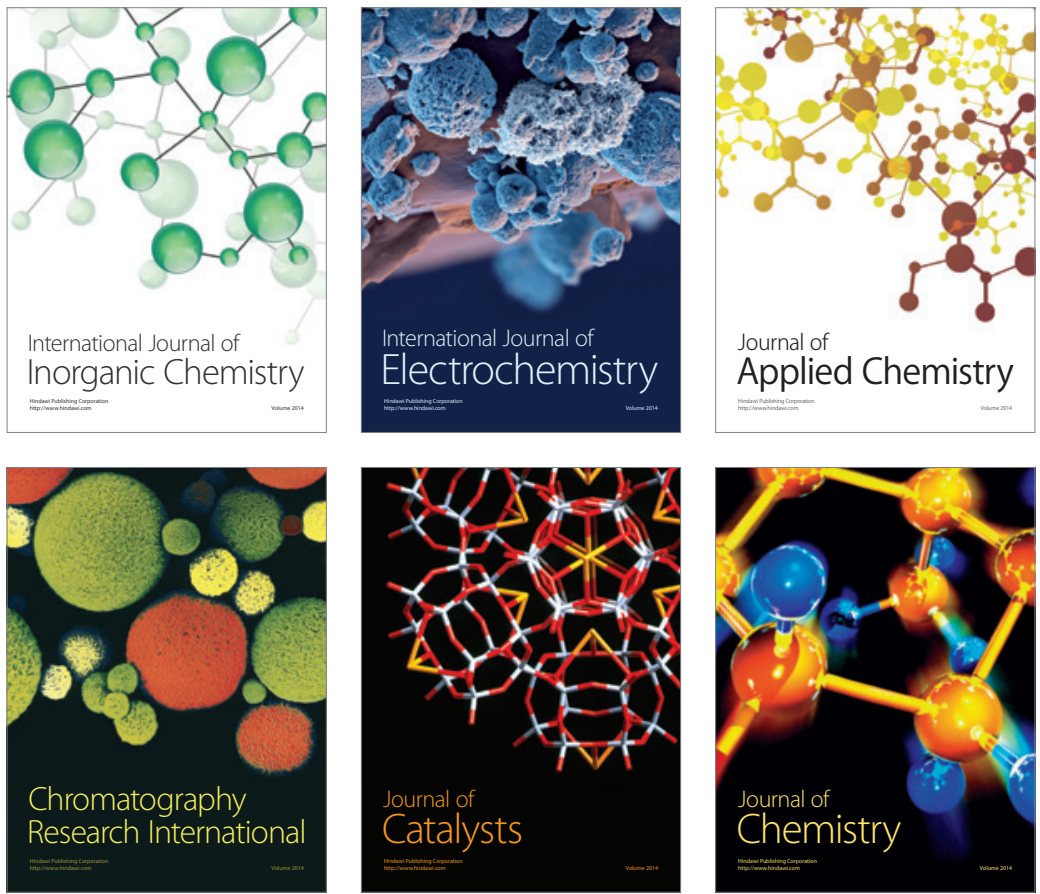

Journal of

Applied Chemistry
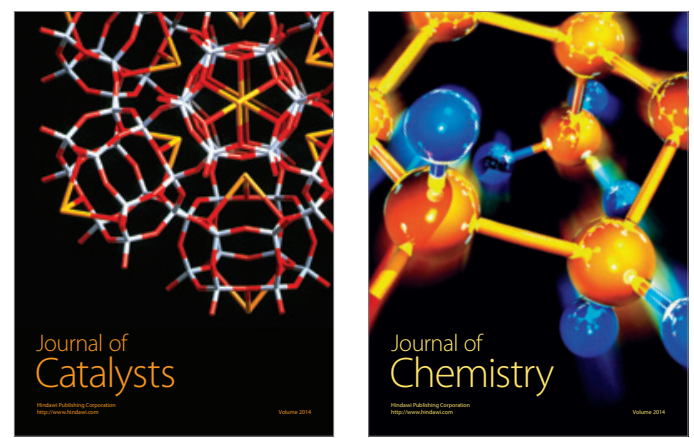
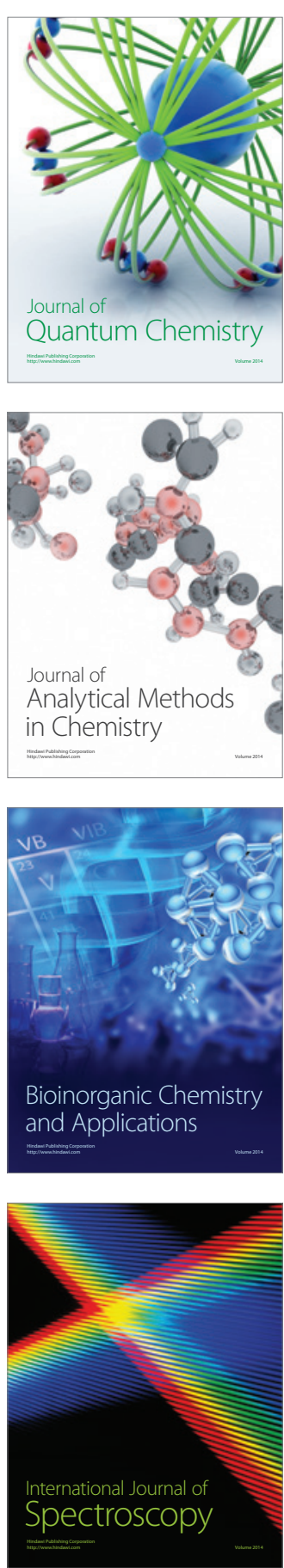